\title{
Research on the simulation evaluation platform for the Ordnance soldier's professional skill appraisal
}

\author{
Rongyue Xie ${ }^{1, a}$,Zhiyong cheng ${ }^{1, b}$, Xiangjun $\mathrm{Xu}^{1, \mathrm{c}}$, Zhongtao Zhu ${ }^{1, \mathrm{~d}}$ Qiu lei ${ }^{1, \mathrm{e}}$ \\ Zhou xiao ${ }^{1, \mathrm{f}}$ \\ ${ }^{1}$ Wuhan Mechanical Technology College, Wuhan, 430075, China \\ aemail: ryxie@126.com, bemail: \\ zycheng72@sina.com, cemail:xiangjunxu@163.com, 'email:zhongtaozhu@163.com, \\ eemail: hnnhlxy@163.com, ${ }^{\mathrm{f} e m a i l}$ : leopzhx@sohu.com
}

Keywords: Ordnance; Occupation skill appraisal; Simulation assessment; Platform

\begin{abstract}
Based on the current situation that the ordnance soldiers are lacking a complete set of professional skill appraisal assessment data, the present on the guarantee difficulty for live-fire training, a new simulation assessment platform was designed and realized in this paper, which included the theory examination and virtual interactions examination. This system can well compatible with the existing paperless examination system platform. Through studying the key technologies such as opening examination and virtual interaction, the Simulation virtual examination platform was realized, which provides a new digital method for the organizers and students.
\end{abstract}

\section{Introduction}

With the advancement of military professional skill appraisal work, because of the lack of supporting information in the army, operation assessment training guarantee difficulty was becoming harder and harder. To improve the soldier professional skills and improve identification station inspection organization, it urgently needs to reform the existing way of training and assessment. Development of ordnance soldiers' professional skill appraisal simulation test platform became reality demand.

In this paper, the theory examination based on random algorithm and the open virtual operating examination unified organically in together. The paperless examination of basic theory and virtualization for the live-fire operation were realized. It provided a system of complete set of data form and simulation platform for the troop's skill appraisal organizer and the examinees. What's more, it provided a new digital method of training for the professional skill appraisal work.

\section{Institutions Optimization Design}

\section{A. Approaches of General Scheme}

Visual Studio 2010 was adopted as system integrated development environment in this paper. Access was used as the database engine, and VIRTOOL was used as virtual operation interactive simulation engine. In order to fit different requirements, two kinds of architecture were adopted in the system. The first one is four layers architecture for virtual simulation, mainly used in basic skills and professional simulation training and evaluation section. The other is three-layer architecture for theoretical module. It is better able to distinguish the function of each layer by using the advantage of multi-tier architecture. It also provided the convenient design and maintenance. The system has good scalability and flexibility. The diagram of the framework is shown as Fig. 1. 

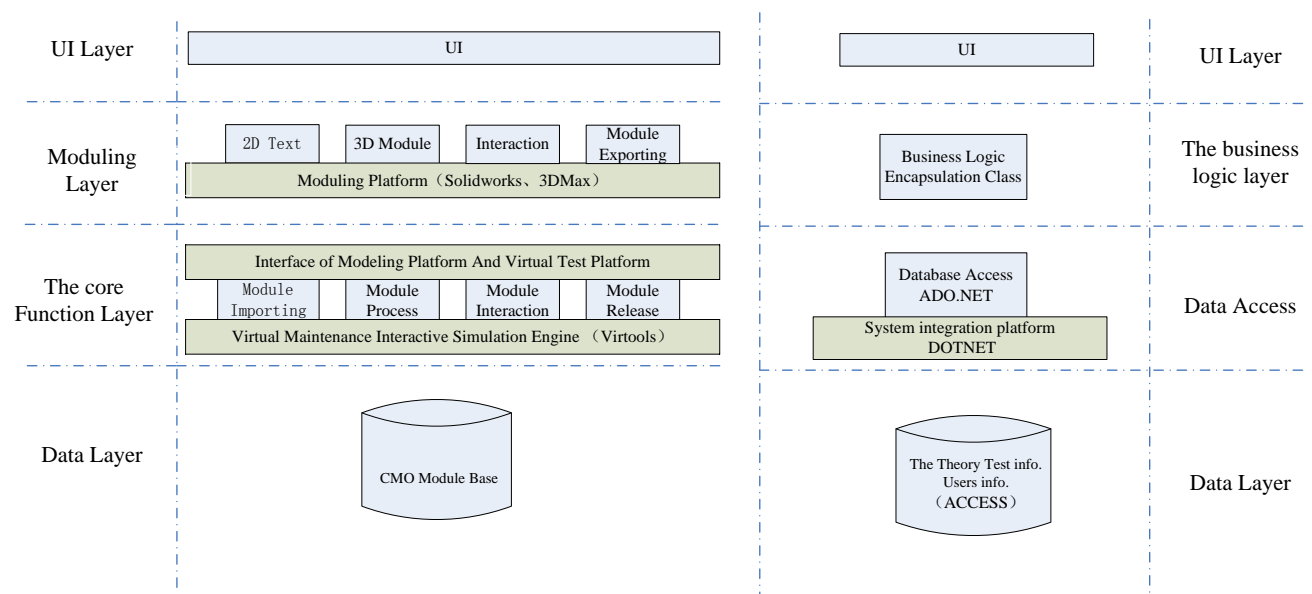

\section{B. System Composition}

Fig.1. Diagram of Virtual simulation platform architecture

According to the simulation assessment and training platform development requirements, three basic function modules, including the theory module, basic skills, professional skills module, were designed for different professional soldiers' assessment of professional skill appraisal personnel requirements in this system. The function modules corresponds to the current ordnance equipment support professional skill appraisal outline the inspection requirements. It completely covered the current appraisal ways. The block diagram of the system is shown as Fig. 2.

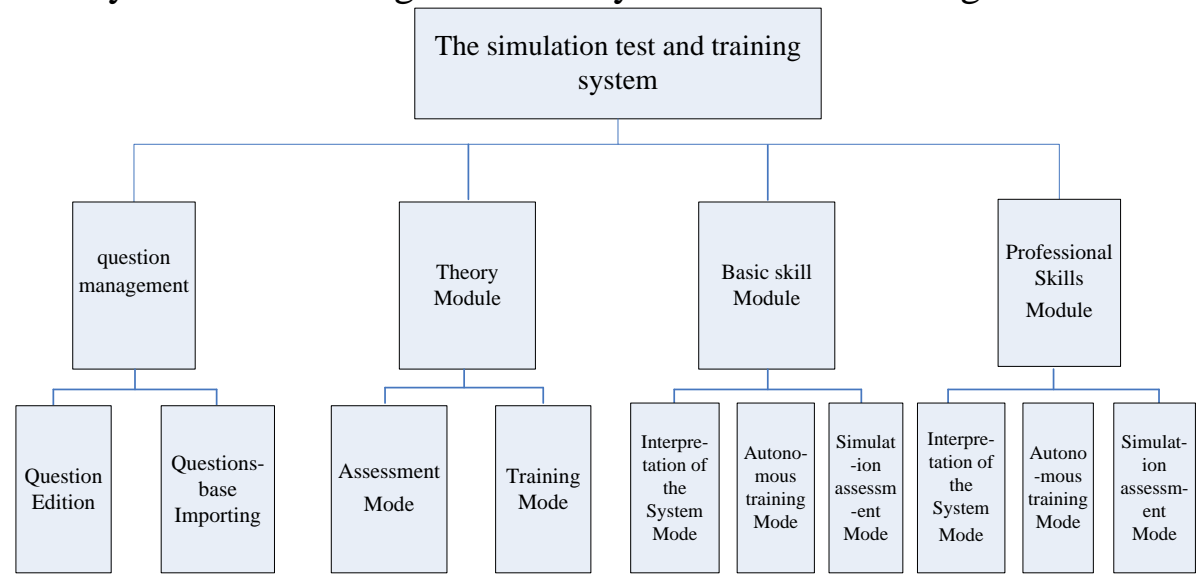

Fig.2. Block diagram of the simulation test and training system

\section{The Specific Contents of the System}

\section{A. The Software System Platform Research}

In ordnance soldiers mock exams and training of professional skill appraisal system platform, the realization of basic skills and professional skills operation virtualization is one of the core content, which is also the difficulty. It became the key problem to solve in this system, which provides the virtual environment in which operation with good interactive performance could be completed or not.

Fusing high quality 3D model of the operating units to a series of pre-defined action process, and calling through interactive virtual engine, it provided users with a realistic operating environment in this paper. The flow chart of building software system platform building is shown as Fig. 3. 


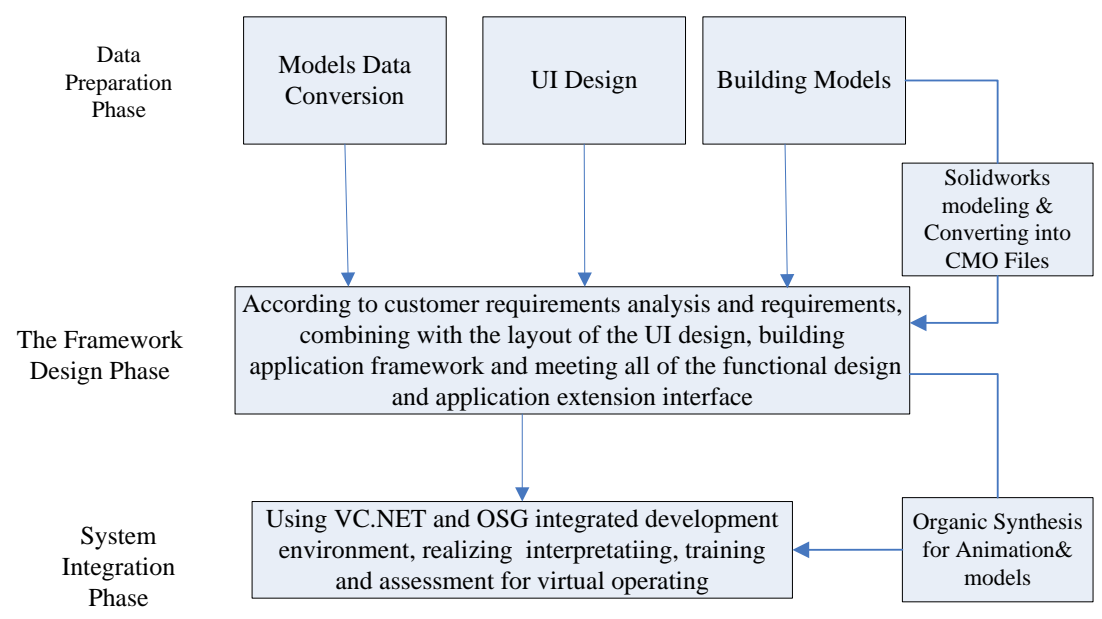

Fig.3. Chart of building software system platform

\section{B. The realization of the virtual simulation training and simulation evaluation}

Virtual simulation training and simulation evaluation of interactive operation was the core of the whole simulation module, and the key to the simulation test and training system.

Users achieve a goal of training through virtual interaction operation procession. After importing the models, the definition of virtual operating interactive process was completed by the behavior engine and rendering engine for module generated, and interaction module was released by release of model. The flow chart of implementation process of virtual interactions in the VIRTOOL is shown as Fig.4.

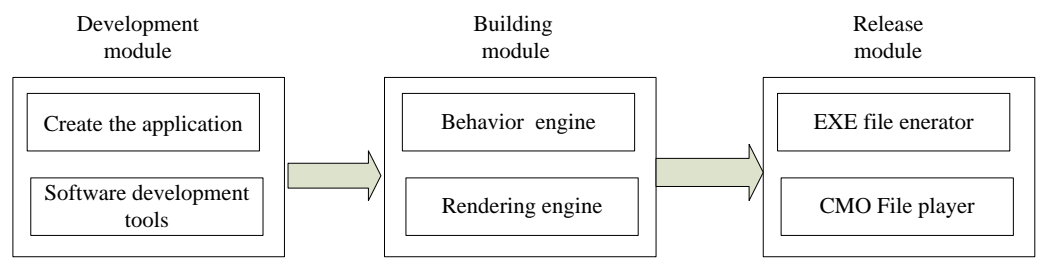

Fig.4. Chart of implementation process for virtual interactions in VIRTOOL

\section{The design for the dynamic process analysis module}

This module design is mainly due to the different subjects in a variety of forms of professional skill examination. Some subjects are abstract, and it is not easy to express by virtual means such as 3D model. A dynamically dragging flow chart way is proposed in this paper to show such subjects. Video, animation, and graphic auxiliary instructions were added into each procession node, which can improve the practicability and validity of subject training.

\section{The design of the theoretical examination module}

This system is developed by the idea of systematic and modular design. In this theoretical examination module, it can be read directly by that the user selected professional, grade and examination mode. Users can reach the specified mode, and can take examination online according to certain rules, train independently using the questions related professional and levels.

\section{Key Techniques of the System}

\section{A. The design of random question selection algorithm}

Currently, many test adopted electronic test system to ensure privacy and fairness in the exam. The basic idea is that questions are drawn randomly according to the preset knowledge from during the test questions to compose of a set of test paper. In order to guarantee the generated test paper to vary each time, it demands that every time exam must be randomly drawn from the question bank. How to do item selection of randomness? It involves the use of random function. In this paper, the function random (Int32) in C\# language is used to generate Random Numbers, using unchecked parameter ((Int)DateTime.Now.Ticks) as the incoming seeds.

\section{B. The design of open test}

Virtual simulation method is used in the skill examination. As virtual simulation equipment is 
not really practical equipment, its operation is difficult to achieve the live-fire one. Namely open operation is hard to be realized. If you want to do open operation, you need to equip the logic function of the virtual simulation. The electrician experiment of this system is designed by this full open idea in the virtual examination.

\section{System integration}

The software platforms in this system involve modeling platform, virtual operating interactive engine, database platform and so on, so it need to be integrated in .Net platform by making full use of the interface provided by the software. In the process of integration, the main problem considered is the manipulation of CMO file. The solution is shown bellow. The CMO file displays by calling the VT player controls in .Net platform system. But controlled the model is achieved by mouse and keyboard. The control can be directly manipulate through the keyboard and mouse in the focused cases. So operation of the CMO file can be realized only needing that the keyboard and mouse events are realized on the system platform. The system main interface is shown as Fig. 5 .

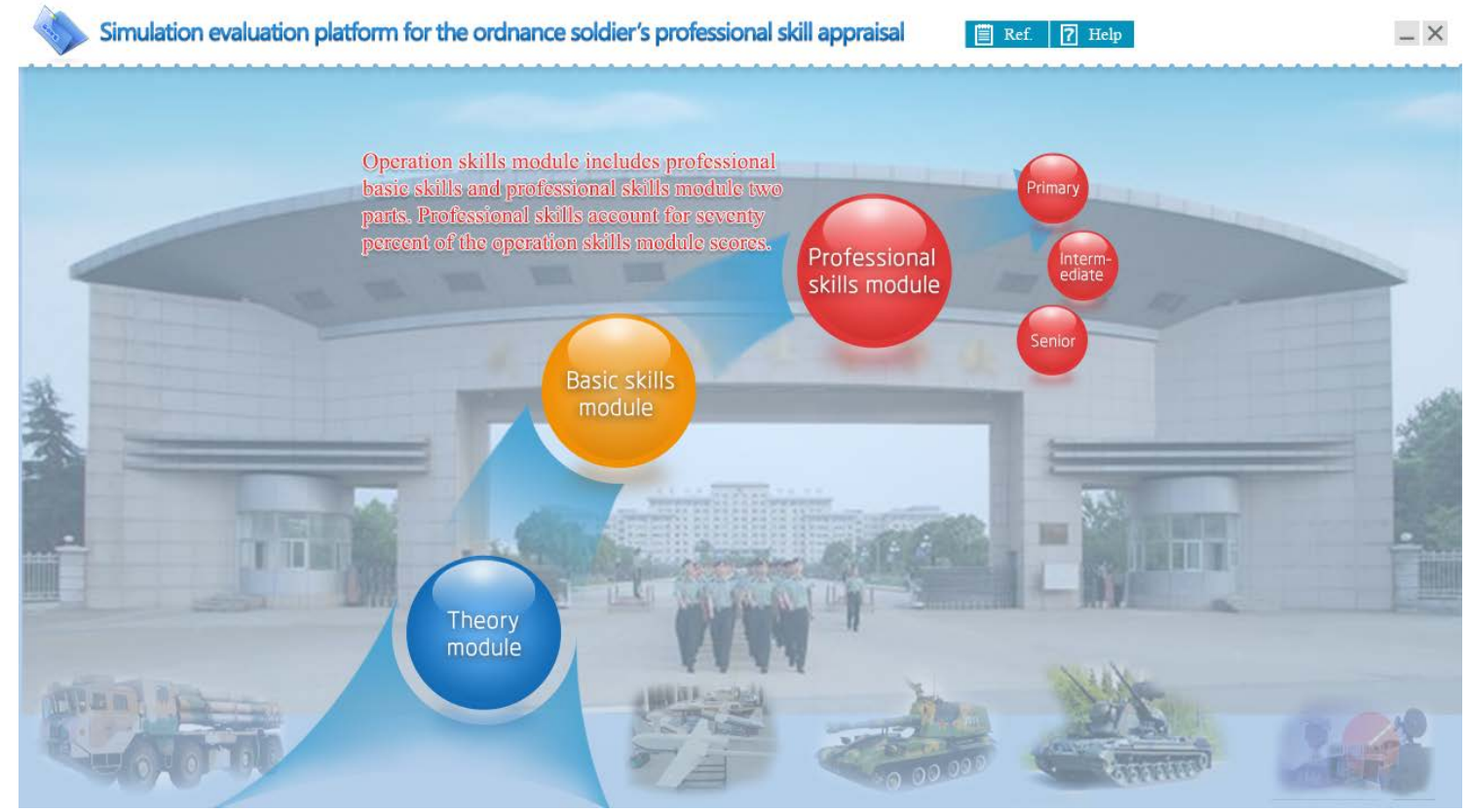

Fig.5. Main interface of the system

\section{Summary}

Through the development of the simulation evaluation platform for the Ordnance soldier's professional skill appraisal, it meets the new demand of relevant personnel, and improvs the level and quality of soldiers identification. At the same time, this system as a general platform for ordnance soldier skill appraisal training has portability, scalability, and scalability. It can be referred by the other general equipment support professionals. It is important for the development of professional skill appraisal work.

\section{References}

[[1] Cheng Hongfang,Zhang Dengfeng, Li Gaomin. Research on Effectiveness of Visual System Based on Flight Engineering Simulator[J]. Journal of System Simulation, 2006.18(2):441 443( in Chinese)

[2] Li Jun, Wang Shaodi,Chang Jiangang, Sun Zhixin,Wang Ruchuan.Analysis and Design of Rendering Software Based on Vega[J]. Journal of System Simulation.2003,15(3):397 400(in Chinese)

[3] Xu Dongping, Zhong Tao, Zhou Shisheng. Research on Framwork for Real-Time Interactive 
Scene Simulation System[J]. Journal of Wuhan University of Technology,2004,28(6):930 932(in Chinese)

[4] Linyi, A Novel Software Architecture for Visualization Simulation[J], JOURNAL OF NORTHWESTERN POLYTECHNICAL UNIVERSITY, 2008 26(2):184 188

[5] Shuchang. Research on an Equipment Visual Emluator Based on 3Dmax and OGRE[J], Command Control \& Simulation, 2009.31(5):78 80.

[6] G Antonino, Zachmann Gabriel. Virtural reality as a tool for verification of assembly and maintenance process [J]. Computer and Graphics ,1999, 23(3):389 403. 